\title{
Spectral and timing properties of the accreting X-ray millisecond pulsar IGR J17511-3057
}

\author{
M. Falanga ${ }^{1}$, L. Kuiper ${ }^{2}$, J. Poutanen ${ }^{3}$, D. K. Galloway ${ }^{4}$, E. W. Bonning ${ }^{5}$, E. Bozzo ${ }^{6}$, A. Goldwurm ${ }^{7,8}$, \\ W. Hermsen ${ }^{2,9}$, and L. Stella ${ }^{10}$ \\ ${ }^{1}$ International Space Science Institute (ISSI), Hallerstrasse 6, 3012 Bern, Switzerland \\ e-mail: mfalanga@issibern.ch \\ 2 SRON - Netherlands Institute for Space Research, Sorbonnelaan 2, 3584 CA, Utrecht, The Netherlands \\ 3 Astronomy Division, Department of Physics, PO Box 3000, 90014 University of Oulu, Finland \\ 4 School of Physics and School of Mathematical Sciences, Monash University, VIC 3800, Australia \\ 5 Department of Physics and Yale Center for Astronomy and Astrophysics, Yale University, PO Box 208121, New Haven, \\ CT 06520-8121, USA \\ ${ }^{6}$ ISDC, Data centre for astrophysics, University of Geneva, Chemin d'Écogia 16, 1290 Versoix, Switzerland \\ 7 Service dAstrophysique (SAp), IRFU/DSM/CEA-Saclay, 91191 Gif-sur-Yvette Cedex, France \\ ${ }^{8}$ Unité mixte de recherche Astroparticule et Cosmologie, 10 rue Alice Domon et Leonie Duquet, 75205 Paris, France \\ 9 Astronomical Institute “Anton Pannekoek”, University of Amsterdam, Science Park 904, 1098 XH, Amsterdam, The Netherlands \\ 10 INAF - Osservatorio Astronomico di Roma, via Frascati 33, 00040 Monteporzio Catone, Roma, Italy
}

Received 1 December 2010 / Accepted 23 January 2011

\begin{abstract}
Context. IGR J17511-3057 is the second X-ray transient accreting millisecond pulsar discovered by INTEGRAL. It was in outburst for about a month beginning on September 13, 2009.

Aims. We analyze the spectral and timing properties of the object and the characteristics of X-ray bursts with the aim to constrain the physical processes responsible for the X-ray production in this class of sources.

Methods. We studied the broad-band spectrum of the persistent emission in the $0.8-300 \mathrm{keV}$ energy band using simultaneous INTEGRAL, RXTE and Swift data obtained in September 2009. We also describe the timing properties in the 2-120 keV energy range such as the outburst light curve, pulse profile, pulsed fraction, pulsed emission, and time lags, and moreover study the properties of X-ray bursts discovered by RXTE and INTEGRAL and the recurrence time.

Results. The broad-band average spectrum is well described by thermal Comptonization with an electron temperature of $k T_{\mathrm{e}} \sim$ $25 \mathrm{keV}$, soft seed photons of $k T_{\mathrm{bb}} \sim 0.6 \mathrm{keV}$, and Thomson optical depth $\tau_{\mathrm{T}} \sim 2$ in a slab geometry. During the outburst the spectrum stays remarkably stable with plasma and soft seed photon temperatures and scattering optical depth being constant within errors. We fitted the outburst profile with the exponential model, and using the disk instability model we inferred the outer disk radius to be $(4.8-5.4) \times 10^{10} \mathrm{~cm}$. The INTEGRAL and RXTE data reveal the X-ray pulsation at a period of 4.08 milliseconds up to $\sim 120 \mathrm{keV}$. The pulsed fraction is shown to decrease from $\sim 22 \%$ at $3 \mathrm{keV}$ to a constant pulsed fraction of $\sim 17-18 \%$ between $7-30 \mathrm{keV}$, and then to decrease again to $\sim 13 \%$ at $60 \mathrm{keV}$. The nearly sinusoidal pulses show soft lags that monotonically increase with energy to about $0.2 \mathrm{~ms}$ at $10-20 \mathrm{keV}$ similar to those observed in other accreting pulsars. The short burst profiles indicate hydrogen-poor material at ignition, which suggests either that the accreted material is hydrogen-deficient, or that the CNO metallicity is up to a factor of 2 times solar. However, the variation of the burst recurrence time as a function of $\dot{m}$ (inferred from the X-ray flux) is much smaller than predicted by helium-ignition models.
\end{abstract}

Key words. pulsars: individual: IGR J17511-3057 - stars: neutron - X-rays: binaries - X-rays: bursts

\section{Introduction}

The discovery of radio millisecond pulsars in binary systems in the 1970s lead to the prediction that neutron stars hosted in low-mass X-ray binary systems (LMXB) were their progenitors (Alpar et al. 1982). As a binary system evolves through phases of accretion onto the neutron star (NS), it gains angular momentum from the accreted material, which is sufficient to spin-up the NS to a rotation period equilibrium in the millisecond range. The first confirmation that LMXBs can host rapidly rotating NSs was the discovery of coherent oscillations from type-I X-ray bursts (see Strohmayer \& Bildsten 2006, for a review). Later identification of SAX J1808.4-3658 as a $401 \mathrm{~Hz}$ pulsar (Wijnands \& van der Klis 1998) led to the discovery of a new class of accreting NSs, accreting millisecond X-ray pulsars (AMXPs). The first direct measurement of spin-up of the NS during an accretion phase was published by Falanga et al. (2005a) in the source IGR J00291+5934, strengthening the hypothesis of the recycling of old radio-pulsars to millisecond periods (see also the reanalysis by Patruno 2010). On the other hand, between the outbursts, long-term monitoring shows some AMXPs to exhibit spin-down in quiescence (Hartman et al. 2009; Patruno 2010; Papitto et al. 2011). The spin frequencies of AMXPs lie in the range of 180 $600 \mathrm{~Hz}$, and the orbital periods are between $40 \mathrm{~min}$ and $5 \mathrm{~h}$ (see reviews by Wijnands 2006; Poutanen 2006).

The AMXPs exhibit many characteristics similar to other LMXBs. Their broadband spectra show soft thermal and hard Comptonized components (see reviews by Poutanen 2006; Falanga 2008) similar to atoll sources in the low/hard state 
(Barret et al. 2000). The AMXPs also show quasi-periodic oscillations and X-ray bursts (see e.g., Chakrabarty et al. 2003; Watts et al. 2005). The pulse profiles are typically close to sinusoidal with modulations of $3-15 \%$. The pulse profiles are energy-dependent and demonstrate soft time-lag in the range up to 100-250 $\mu$ s (e.g., Cui et al. 1998; Ford 2000; Poutanen \& Gierliński 2003; Gierliński \& Poutanen 2005; Falanga \& Titarchuk 2007).

The magnetic field of the NS as inferred from accretion models is relatively weak, in the range of $\sim 10^{8}-10^{9} \mathrm{G}$ (Psaltis \& Chakrabarty 1999; Di Salvo \& Burderi 2003). Measurements of the inner disk radius from evolution of the pulse profiles and the spin-down of the pulsars between the outbursts allow a more accurate determination of the magnetic field of $1.5 \times 10^{8} \mathrm{G}$ in SAX J1808.4-3658 (Ibragimov \& Poutanen 2009; Hartman et al. 2009) and of $2 \times 10^{8} \mathrm{G}$ in IGR J00291+5934 (Patruno 2010).

Pulsations are not detected during quiescence in either X-ray or optical bands, nor has millisecond radio pulsed emission been found (see e.g., Iacolina et al. 2010, and references therein). Following outbursts, the optical counterpart has been observed to diminish in intensity consistent with the X-ray flux decay rate implying the origin of the emission to be in the accretion disk (see e.g., Falanga et al. 2005a). Companion stars of AMXPs are highly evolved white or brown dwarfs (Deloye \& Bildsten 2003). In the AMXPs with an orbital period in the hour range and a hydrogen-rich donor brown dwarf, X-ray bursts have been detected (see e.g., Galloway \& Cumming 2006), during which burst oscillations are nearly in phase with the coherent oscillation at the spin period (Chakrabarty et al. 2003; Strohmayer et al. 2003).

\subsection{The source IGR J17511-3057}

Among roughly one hundred LMXBs hosting a NS, IGR J17511-3057 is the twelfth known to host an AMXP. It is the second source discovered by INTEGRAL during the Galactic Bulge monitoring program (Baldovin et al. 2009) that was found to be an AMXP with RXTE (Markwardt et al. 2009a). The INTEGRAL-derived source position for the new transient was $\sim 20^{\prime}$ away from the known $435 \mathrm{~Hz}$ millisecond pulsar XTE J1751-305, the source position uncertainty of $2^{\prime}$, as well as the subsequent detection of a distinctly different pulse frequency of $244.8 \mathrm{~Hz}$ confirmed the transient to be a new AMXP (Markwardt et al. 2009a). The orbital period of IGR J17511-3057 is $\sim 3.47 \mathrm{~h}$, with an $a \sin (i) / c$ value of $\sim 275.2$ lt-ms (Riggio et al. 2011, and references therein). A candidate near-infrared counterpart was found by Torres et al. (2009a) on September 22, 2009 with a $K_{\mathrm{s}}$-band magnitude of $18.0 \pm 0.1$, which had faded to $K_{\mathrm{s}}>18.8$ ( $3 \sigma$ upper limit) by October 7, 2009 (Torres et al. 2009b). Radio upper limits of 0.16-0.18 mJy between September 16 and 25, 2009 were set with the VLA by Miller-Jones et al. (2009). The most accurate position of the source was provided by the near-infrared observations at $\alpha_{\mathrm{J} 2000}=17^{\mathrm{h}} 51^{\mathrm{m}} 08^{\mathrm{s}} .64$ and $\delta_{\mathrm{J} 2000}=-30^{\circ} 57^{\prime} 40^{\prime} \cdot 70$ (Torres et al. 2009a), consistent with the $1 \sigma$ error of 0.6 of the Chandra/HETG position (Nowak et al. 2009).

The first type-I X-ray burst from the source was detected by Swift (Bozzo et al. 2009), and the Swift monitoring of this source in outburst was reported by Bozzo et al. (2010), who found no evidence for photospheric radius expansion during the three studied bursts. All follow-up observations with different spacecrafts detected X-ray bursts from the source. Burst oscillations at $\sim 245 \mathrm{~Hz}$ were first detected in an X-ray burst on September 14, 2009 by RXTE/PCA (Watts et al. 2009); $X M M-N e w t o n$ also detected X-ray bursts with the burst oscillations phase locked to the persistent pulsations (Papitto et al. 2009). A Chandra observation on September 22, 2009 also detected an X-ray burst (Nowak et al. 2009). The net persistent spectrum, 0.5-10 keV band, of IGR J17511-3057 was found for all the observations to be well-fit by an absorbed powerlaw model with photon index $\sim 2$ and column density $N_{\mathrm{H}}=$ (0.6-1.3) $\times 10^{22} \mathrm{~cm}^{-2}$, depending on the model used (Bozzo et al. 2009; Nowak et al. 2009; Papitto et al. 2010a).

A lower limit on the companion mass of $0.13 M_{\odot}$ was determined by Markwardt et al. (2009a) using the mass function of the system and assuming a NS mass of $1.4 M_{\odot}$ and an inclination angle of $90^{\circ}$. This lower limit was later improved by Papitto et al. (2010a), who considered that no occultation or dips were observed during the outburst of IGR J17511-3057. These authors also performed a fit to the source spectrum with a reflection continuum model and determined an inclination angle for the system in the range $38-68^{\circ}$. The corresponding limits on the companion mass in this case are $0.15-0.23 M_{\odot}$ (a NS of $1.4 M_{\odot}$ was assumed). We refer the reader to Papitto et al. (2010a) for more details regarding the nature of the companion star. An upper limit on the source distance of $\sim 10 \mathrm{kpc}$ was estimated first by Papitto et al. (2010a) and $7 \mathrm{kpc}$ by Altamirano et al. (2010), assuming that the most energetic type-I X-ray burst observed from IGR J17511-3057 reached the Eddington luminosity.

In this paper we report on INTEGRAL and RXTE observations of IGR J17511-3057, as well as a simultaneous Swift, INTEGRAL, and RXTE observation during the period MJD 55 087-55117 (September 13-October 13, 2009). We study the light curves, broad-band spectra, outburst spectral evolution, and timing properties of the source. The properties of the largest set of X-ray bursts from this source are investigated as well.

\section{Observations and data}

\subsection{INTEGRAL}

The present data were obtained during the INTEGRAL (Winkler et al. 2003) Target of Opportunity (ToO) observation during satellite revolution 846, beginning on September 16 (MJD 55090.92557) and ending on September 19, 2009 (MJD $55093.58993)$, with a total net exposure time of $206 \mathrm{ks}$. The data reduction was performed using the standard Offline Science Analysis $\left(\mathrm{OSA}^{1}\right)$ version 8.0 distributed by the Integral Science Data Center (Courvoisier et al. 2003). The algorithms used for the spatial and spectral analysis are described in Goldwurm et al. (2003). The observation aimed at IGR J17511-3057 consists of 70 stable pointings with a source position offset $\$ 7^{\circ} .0$ from the center of the field of view. We analyzed data from the IBIS/ISGRI coded mask telescope (Ubertini et al. 2003; Lebrun et al. 2003) at energies between 18 and $300 \mathrm{keV}$ and from the JEM-X monitor, module 1 (Lund et al. 2003) between 3 and $20 \mathrm{keV}$. Because this observation was in a rectangular pattern model which consists of a square $5 \times 5$ pattern around the nominal target location, IGR J17511-3057 was only within the source position offset of $<3.5$ from the center of the field of view for 28 stable pointings. Therefore, for JEM-X with a field of view of 7.5 (diameter) at half response the effective exposure time was only $87.4 \mathrm{ks}$.

In Fig. 1 we show part of the ISGRI field of view (significance map) centered on the position of IGR J17511-3057 (20$70 \mathrm{keV}$ energy range). The source was clearly detected in the

${ }^{1}$ http://www.isdc.unige.ch/integral/analysis 


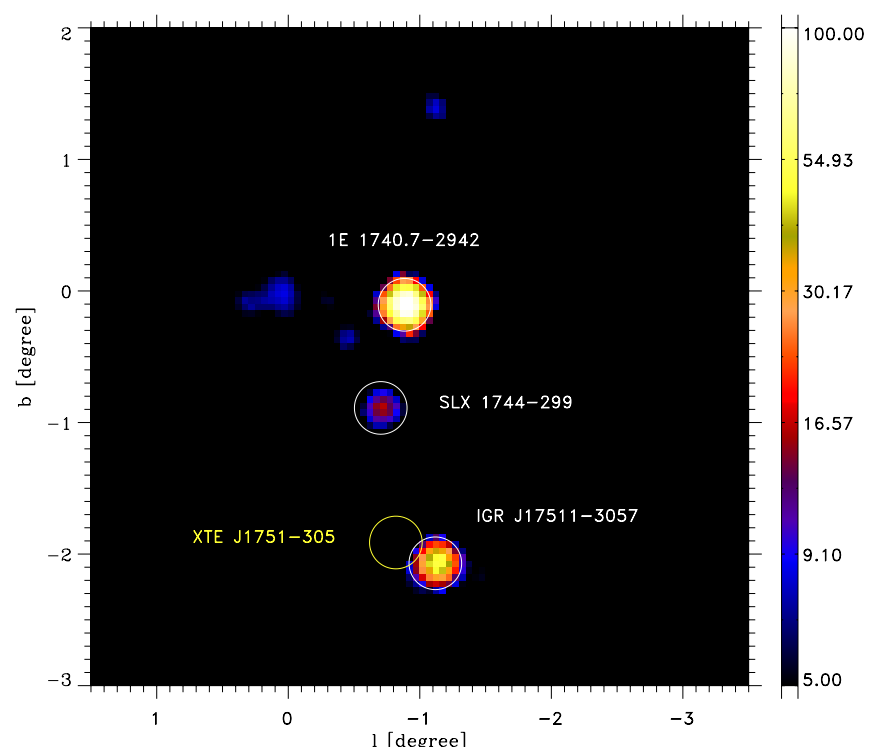

Fig. 1. INTEGRAL/ISGRI sky image of the field of view around IGR J17511-3057 (20-70 keV). The size of each pixel in the image corresponds to $3^{\prime}$. Evidently the non-imaging instruments on-board RXTE with a field of view of $\sim 1^{\circ}$ (radius) are not be able to separate the emission from the two AMXPs XTE J1751-305 and IGR J17511-3057 when they are both active in X-rays (see Fig. 2).

mosaic, and we estimated detection significances of $\sim 65 \sigma$ in the $20-40 \mathrm{keV}$ energy range and $\sim 29 \sigma$ in the $40-100 \mathrm{keV}$ energy range. The best determined position is at $\alpha_{\mathrm{J} 2000}=17^{\mathrm{h}} 51^{\mathrm{m}} 08^{\mathrm{s}} .74$ and $\delta_{\mathrm{J} 2000}=-30^{\circ} 57^{\prime} 36^{\prime \prime}$. 9 , with an associated uncertainty of 0.55 at the $90 \%$ c.l. (20-40 keV, Gros et al. 2003). The offset with respect to the near-infrared position is 0'07 (Torres et al. 2009a). In the same figure we also indicate with a yellow circle the position of the millisecond pulsar XTE J1751-305 that underwent a short period of enhanced X-ray activity during the last part of the outburst decay of IGR J17511-3057 (see Sect. 2.2 and Fig. 2).

To search for X-ray bursts, the ISGRI light curves are calculated from events selected according to the detector illumination pattern for IGR J17511-3057. For ISGRI we used an illumination factor threshold of 0.6 for the energy range $18-40 \mathrm{keV}$; for JEM-X we used the event list of the whole detector in the 3$20 \mathrm{keV}$ energy band.

\section{2. $R X T E$}

We used publicly available data from the proportional counter array PCA (2-60 keV; Jahoda et al. 1996) and the High Energy Xray Timing Experiment HEXTE (15-250 keV, Rothschild et al. 1998) on-board the RXTE satellite. The FWHM of these collimator instruments is $\sim 1^{\circ}$, and no spatial information of the photons exists. IGR J17511-3057 was monitored from September 13 to October 8, 2009 (MJD 55 087.85619-55112.33693) for a total net exposure time of $\sim 455 \mathrm{ks}$ (observation ID 94041). For the light curve analysis we also added the twelve pointings from observation ID 94042 ( $45.2 \mathrm{ks}$ net exposure time; target XTE J1751-305). In this dataset the nearby X-ray millisecond pulsar XTE J1751-305 underwent a short outburst contemporaneous with the latest outburst phases of IGR J17511-3057 (Chenevez et al. 2009; Markwardt et al. 2009b). Figure 1 shows that the angular separation between the two sources is smaller than the field of view of the non-imaging instruments on-board

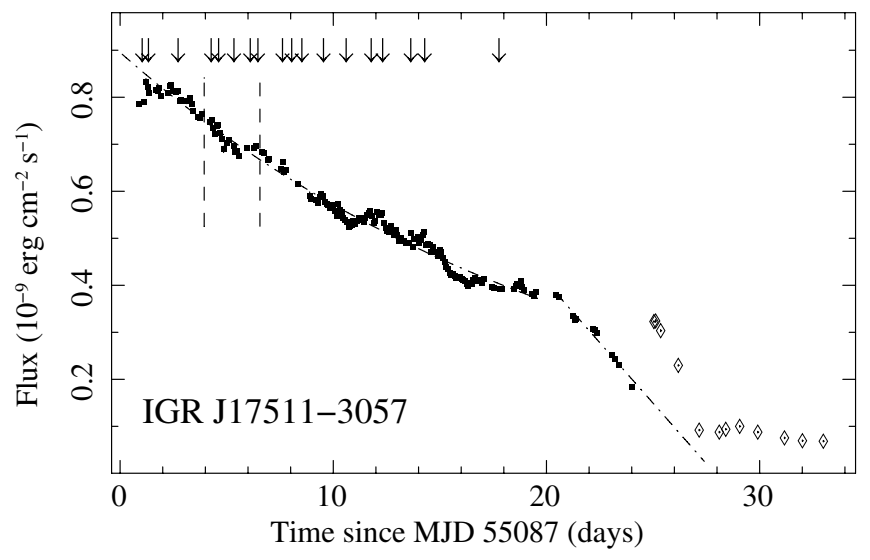

Fig. 2. RXTE/PCA (2-20 keV) outburst light curve of IGR J175113057. For plotting purpose we chose a bin time of $6840 \mathrm{~s}$. The count rate was converted into flux using the spectral results reported in Sect. 4.2. The diamonds correspond to observations in which both XTE J1751305 and IGR J17511-3057 were active and the instruments on-board RXTE were unable to separate the contribution of the two sources. The arrows indicate the times of the detected X-ray bursts (see Sect. 6 and Table 3). The vertical dashed lines indicate the interval of the INTEGRAL observations. The dot-dashed lines correspond to the bestfit exponential profile, $F \propto \mathrm{e}^{-t / 22.4^{\mathrm{d}}}$, and a linear decay.

RXTE $\left(\sim 1^{\circ}\right)$, and thus we were unable to separate the contributions of the two objects from the total X-ray flux.

We carried out a spectral analysis, using Standard-2 data (with $16 \mathrm{~s}$ time resolution) for the PCA and standard Cluster-0 data for HEXTE. For HEXTE, we used the on-source data, using default screening criteria for Cluster 0 . The data were extracted for the Good Time Intervals defined by standard criteria. The PCA response matrix was created by FTOOLS version 6.0.2 for 129 energy channels to cover the energy range from 2-60 keV. For HEXTE we used the standard 64 energy channel response matrix for the $15-250 \mathrm{keV}$ energy range. For the timing analysis we used PCU data, collected in the E_125us_64M_0_1s event mode, recording event arrival times with $122.07 \mu$ s time resolution, and sorting events in 64 PHA channels. Default selection criteria were applied.

\subsection{Swift}

IGR J17511-3057 was also observed with Swift (Burrows et al. 2005) during its outburst in 2009. The results were first published in Bozzo et al. (2010). We used only Swift/XRT data in window-timing mode (WT) that were simultaneously collected with the INTEGRAL observations (i.e. Swift observation ID 00031492005; see Table 1 in Bozzo et al. 2010, for details). We refer the reader to Bozzo et al. (2010) for the Swift XRT data reduction procedure.

\section{Outburst light curve}

Most AMXPs that underwent an outburst for a few weeks to months showed a common outburst profile, i.e. the light curve decays exponentially until it reaches a break, after which the flux drops linearly to the quiescence level (see e.g., Gilfanov et al. 1998; Gierliński \& Poutanen 2005; Falanga et al. 2005b). These outburst profiles were modeled for soft X-ray transients based on the disk instability picture of King \& Ritter (1998), i.e. taking into account the disk irradiation by the central X-ray source during the outburst. King \& Ritter (1998) showed that X-ray 
heating during the decay from outburst causes the light curves of transient LMXBs to exhibit either exponential or linear declines depending on whether or not the luminosity is sufficient to keep the outer disk edge hot. This model has been applied to a sample of different LMXBs, including three AMXPs (Powell et al. 2007). Hartman et al. (2011) investigated the outburst light curve for one of the AMXPs, IGR J00291+5934, using different models in which the decay tail is not necessarily linear. However, Powell et al. (2007) showed that the timescale of the decay light curve and its luminosity at a characteristic time are linked to the outer radius of the accretion disk. A knee in the light curve of the decay from outburst of a transient LMXB is believed to be a consequence of mass transfer onto the outer edge of the disk, because this supply is effectively cut off from the compact object when the outer disk enters the cool low-viscosity state. The X-ray luminosity at which the knee occurs is that at which the outer disk edge is just kept hot by central illumination, allowing this radius to be calculated. In addition, the exponential timescale of the decay gives a second measure of the disk radius.

The 2-20 keV band RXTE light curve of IGR J17511-3057 was extracted for all pointings and is shown in Fig. 2, averaged over $6840 \mathrm{~s}$. The diamonds correspond to the sum of the emission of the X-ray millisecond pulsar XTE J1751-305 in a weak outburst and of IGR J17511-3057 returning back to quiescence. RXTE detected ten and INTEGRAL three type-I X-ray bursts. These are indicated with arrows and are subtracted from the light curve and discussed in Sect. 6. We converted the count rates to flux using the spectral results from Sect. 4.2. The dot-dashed lines correspond to the best-fit exponential profile $F \propto \mathrm{e}^{-t / 22.4^{\mathrm{d}}}$ and a linear decay. In order to fit the light curve of the outburst decay of IGR J17511-3057, we excluded the RXTE data in which the other AMXP XTE J1751-305 was also active. We verified that a linear fit would be probably favored by the data (the reduced $\chi^{2}$ is about half of the corresponding value for the exponential fit). However, given the relatively poor observational coverage over the last part of the outburst decay, a firm distinction between a linear and an exponential decay cannot be made.

From Eqs. (9), (23), and (21) in Powell et al. (2007) it is possible to estimate the outer disk radius in two different and independent ways. Equation (9) gives $L_{\mathrm{X}}=\left(L_{\mathrm{t}}-L_{\mathrm{e}}\right) \exp (-(t-$ $\left.\left.t_{\mathrm{t}}\right) / \tau_{\mathrm{e}}\right)+L_{\mathrm{e}}$, where $L_{\mathrm{e}}, t_{\mathrm{t}}$ (the break time), $L_{\mathrm{t}}$, and $\tau_{\mathrm{e}}$ (exponential decay time) are all free parameters. By using for these parameters the values determined from the fit to the light curve of the outburst decay of IGR J17511-3057 (see Fig. 2), the outer disk radius can be estimated from $R_{\text {disk }}\left(\tau_{\mathrm{e}}\right)=\left(\tau_{\mathrm{e}} 3 v_{\mathrm{KR}}\right)^{1 / 2} \approx$ $4.8 \times 10^{10} \mathrm{~cm}$. Here we adopted for the viscosity near the outer disk edge, $v_{\mathrm{KR}}=4 \times 10^{14} \mathrm{~cm}^{2} \mathrm{~s}^{-1}$, in agreement with King \& Ritter (1998) and Powell et al. (2007). An independent estimate of $R_{\text {disk }}$ can also be obtained by using Eq. (21) in Powell et al. (2007), i.e. $R_{\text {disk }}\left(L_{\mathrm{t}}\right)=\left(\Phi L_{\mathrm{t}}\right)^{1 / 2}$. By assuming $\Phi \approx 1.3 \mathrm{~cm}^{2} \mathrm{~s} \mathrm{erg}^{-1}$ (see Powell et al. 2007), we obtained $R_{\text {disk }}\left(L_{\mathrm{t}}\right) \approx(4.8-5.4) \times 10^{10} \mathrm{~cm}$, for a distance to the source of 6.3 and $7 \mathrm{kpc}$, respectively (see also Sect. 6). Note that the two independent estimates of the inner disk radius agree remarkably well if a distance to the source of $6.3 \mathrm{kpc}$ is considered. A similar agreement was also found for a number of different AMXPs, see Powell et al. (2007). This radius also fulfills the condition $R_{\text {circ }}<R_{\text {disk }}<b_{1}$, where $R_{\text {circ }} \approx(2.8-2.3) \times 10^{10} \mathrm{~cm}$ is the circularization radius and $b_{1} \approx(6.7-6.4) \times 10^{10} \mathrm{~cm}$ is the distance of the Lagrange point $L_{1}$ from the center of the neutron star (see e.g., Frank et al. 2002). To estimate these values we used a companion star mass between $0.15-0.23 M_{\odot}$ (Papitto et al. 2010a), for a NS mass of $1.4 M_{\odot}$.

\section{Spectral analysis}

The spectral analysis was carried out using XSPEC version 12.6 (Arnaud 1996). For the contemporaneous data we combined the 3-22 keV RXTE/PCA data, and the 5-300 keV INTEGRAL/JEM-X/ISGRI data taken on September 16-19, 2009. In addition, for the low-energy range $0.5-10 \mathrm{keV}$ we also used the contemporaneous Swift data (ID 00031492005 WT) from Bozzo et al. (2010). For each instrument, a multiplication factor was included in the fit to take into account the uncertainty in the cross-calibration of the instruments. For all fits the factor was fixed at 1 for the ISGRI data. To follow the outburst spectra outside the INTEGRAL observation interval we used the RXTE/PCA/HEXTE data. To take into account contamination by the Galactic ridge emission, we used as background for the PCA spectral fitting the RXTE/PCA data collected when both pulsars were at the lowest X-ray emission level $(6.3 \mathrm{cts} / \mathrm{s})$ (see also Papitto et al. 2010a; Altamirano et al. 2010). All uncertainties in the spectral parameters are given at a $90 \%$ confidence level for single parameter.

\subsection{Broad-band spectrum}

We studied in detail the broad-band X-ray spectrum of IGR J17511-3057 in the energy range 0.8-300 keV, using the joint INTEGRAL/JEM-X/ISGRI, RXTE/PCA, and Swift/XRT data. For the RXTE data we removed the time intervals corresponding to the bursts. We first fitted the joint XRT/JEM-X/PCA/ISGRI (0.8-300 keV) spectrum with a simple photoelectrically-absorbed power-law model, PL, model which was found to be inadequate with a $\chi^{2} /$ d.o.f. $=417 / 141$. A better fit was obtained by replacing the PL with the exponentially cutoff power-law model, cutofFPL, $\left(\chi^{2} /\right.$ d.o.f. $\left.=115 / 140\right)$. We estimated a power-law photon index of $\Gamma=1.61 \pm 0.04$ and a cutoff energy of $E_{\mathrm{c}}=58 \pm 7 \mathrm{keV}$. The absorption was found to be $N_{\mathrm{H}}=0.96_{-0.09}^{+0.10} \times 10^{22} \mathrm{~cm}^{-2}$, which is close to the Galactic value reported in the radio maps of Dickey \& Lockman (1990). Adding a multi-temperature disk blackbody model, DISKBB, to the fit in order to include the contribution of the soft thermal disk emission in the total X-ray flux (Mitsuda et al. 1984) only marginally improved to the fit $\left(\chi^{2} /\right.$ d.o.f. $\left.=105 / 146\right)$. For this spectral component we obtained an inner disk temperature of $k T_{\text {in }}=0.13 \pm 0.02$ and an inner radius of $R_{\text {in }} \sim 601 \sqrt{\cos i} \mathrm{~km}$, in agreement with the results reported by Bozzo et al. (2010). The best-fit value of $R_{\text {in }}$ suggests a relatively high value of the inner disk radius that is hardly compatible with the idea that in AMXP sources the accretion disk extends to a region very close to the NS surface (i.e., $10 \mathrm{~km}$ ). A better description of the emission from IGR J17511-3057 at the lower energies ( $<2 \mathrm{keV}$ ) was obtained by using the higher spectral resolution of the instruments onboard XMM-Newton, as discussed in Papitto et al. (2010a).

In order to compare the IGR J17511-3057 spectrum with previously observed broad-band spectra of the same source class (e.g., Gierliński et al. 2002; Gierliński \& Poutanen 2005; Falanga et al. 2005a,b, 2007; Ibragimov \& Poutanen 2009), we replaced the cutoff power-law model with the thermal Comptonization model, comprs, in the slab geometry (Poutanen $\&$ Svensson 1996). The main model parameters are the Thomson optical depth $\tau_{\mathrm{T}}$ across the slab, the electron temperature $k T_{\mathrm{e}}$, the temperature $k T_{\mathrm{bb}}$ of the soft-seed blackbody photons assumed to be injected from the bottom of the slab, the emission area $A_{\mathrm{bb}}$, and the inclination angle $\theta$ between the slab normal and the line of sight. The best-fit parameters of all models used to fit the data are reported in Table 1. The marginal discrepancy in flux is 
M. Falanga et al.: Accreting X-ray millisecond pulsar IGR J17511-3057

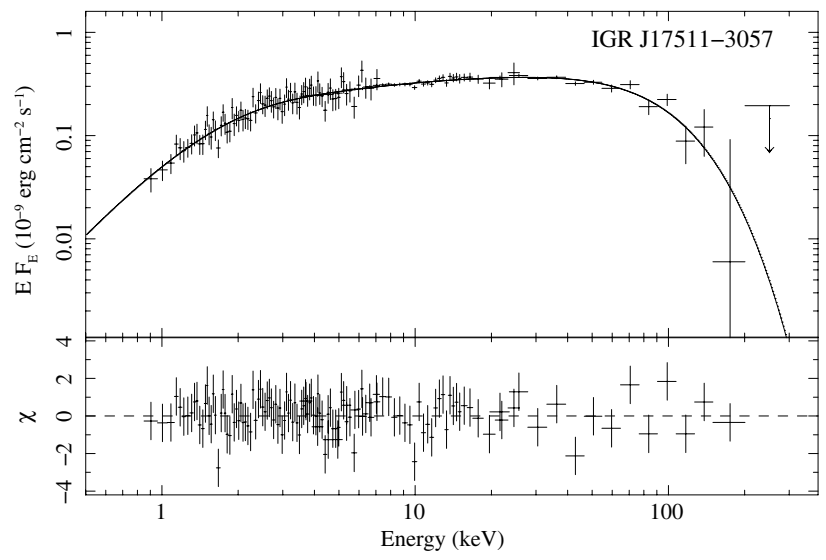

Fig. 3. Unfolded broad-band spectrum of IGR J17511-3057 fit with the thermal Comptonization model comprs. The data points are from XRT (0.8-7 keV), PCA (3-22 keV), and ISGRI (20-300 keV) spectra, respectively. The total model spectrum is shown by a solid line. The lower panel shows the residual between the data and the model.

Table 1. Best-fit spectral parameters of the cutoff power-law and comprs models to the XRT/PCA/ISGRI data.

\begin{tabular}{lcc}
\hline \hline & CUTOFFPL & COMPPS \\
\hline$N_{\mathrm{H}}\left(10^{22} \mathrm{~cm}^{-2}\right)$ & $0.96_{-0.09}^{+0.10}$ & $0.6_{-0.16}^{+0.14}$ \\
$k T_{\mathrm{e}}(\mathrm{keV})$ & - & $24.8_{-2.3}^{+2.4}$ \\
$k T_{\mathrm{bb}}(\mathrm{keV})$ & - & $0.59_{-0.10}^{+0.08}$ \\
$\tau_{\mathrm{T}}$ & - & $2.2_{-0.17}^{+0.14}$ \\
$A_{\mathrm{bb}}{ }^{a}\left(\mathrm{~km}^{2}\right)$ & - & $260_{-90}^{+80}$ \\
$\cos \theta$ & - & $0.53_{-0.10}^{+0.09}$ \\
$\Gamma$ & $1.60_{-0.04}^{+0.04}$ & - \\
$E_{\mathrm{c}}(\mathrm{keV})$ & $58.3_{-6.5}^{+8.1}$ & - \\
$\chi^{2} /$ d.o.f. & $112 / 140$ & $106 / 138$ \\
$F_{\mathrm{bol}}{ }^{b}\left(10^{-9} \mathrm{erg} \mathrm{cm}^{-2} \mathrm{~s}^{-1}\right)$ & $1.41 \pm 0.10$ & $1.28 \pm 0.11$ \\
\hline
\end{tabular}

Notes. ${ }^{(a)}$ Assuming a source distance of 7 kpc. ${ }^{(b)}$ Unabsorbed flux in the $0.8-300 \mathrm{keV}$ energy range.

caused by the difference in the absorption column density measured from the fits with the two spectral models. Below we use $N_{\mathrm{H}}=0.6 \times 10^{22} \mathrm{~cm}^{-2}$, as obtained from the fit with the comprs model. In Fig. 3 we show the unfolded spectrum and the best-fit comprs model. The residuals from the fit are also shown. In this fit, the normalization of the ISGRI data was fixed to 1 as a reference, while the normalizations of the XRT and PCA data were $1.02 \pm 0.08$ and $1.05 \pm 0.05$, respectively. A reasonable value for the normalization of the RXTE PCA could be obtained only after the Galactic ridge emission was taken into account in the background correction of thess data.

\subsection{Spectral evolution during outburst}

We analyzed all 58 RXTE/PCA/HEXTE (3-200 keV) spectra observed during the outburst. We excluded the last twelve RXTE pointings that were contaminated with the emission from a short weak outburst of XTE J1751-305 (see Figs. 1, 2, and Sect. 3). In Fig. 4 we show the best-fit results using the thermal Comptonization comprs model. The burst intervals were removed. For the comprs model the absorption column density and the inclination angle, $\theta$, were fixed at the best-fit value found for

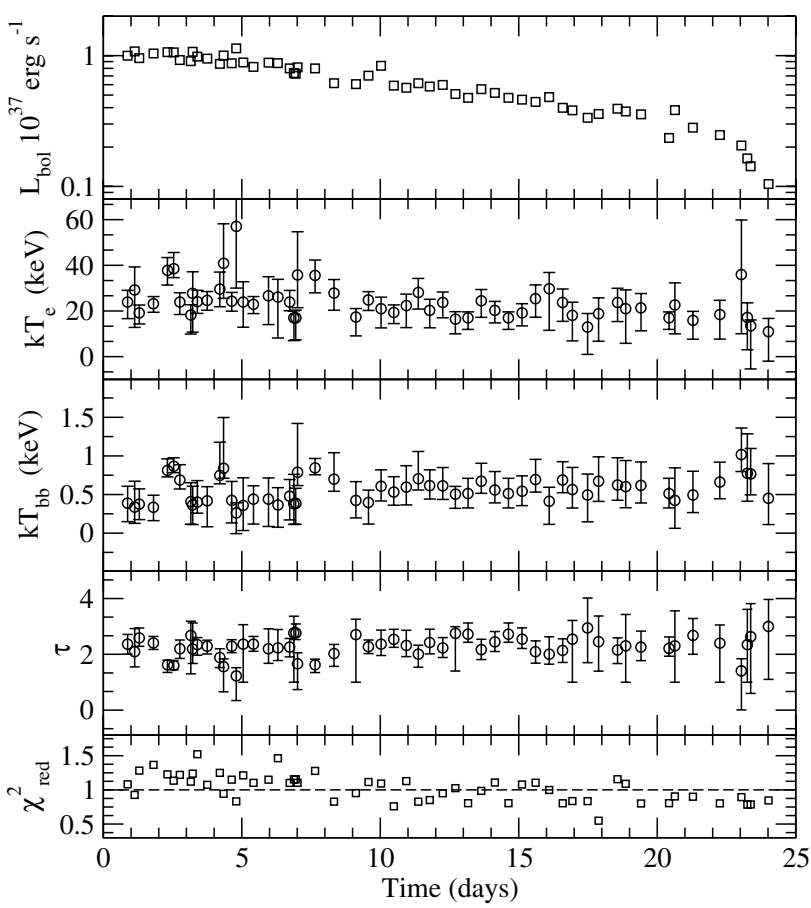

Fig. 4. Outburst evolution of the best-fit spectral parameters of the COMPPs model using the RXTE/PCA/HEXTE data in the 2.5-200 keV range. Each spectrum corresponds to one RXTE observation of IGR J17511-3057. The bolometric luminosity is given in the energy range $0.5-200 \mathrm{keV}$ assuming a source distance of $7 \mathrm{kpc}$.

the broad-band fit (see Table 1 ). The luminosity $L_{\text {bol }}$ was calculated for a distance of $7 \mathrm{kpc}$ (see Sect. 6) from the flux measured for the best-fit model in the energy range $0.5-200 \mathrm{keV}$. These results show that the decay of the outburst is marked by a nearly constant plasma temperature, $k T_{\mathrm{e}}$, soft seed photons emission, $k T_{\text {bb }}$, and optical depth, $\tau$. No statistically significant variations are measured.

A similar behavior, althrough less evident owing to less observational coverage during the outburst, was also observed for the AMXP IGR J00291+5934 (Falanga et al. 2005a). Note that IGR J17511-3057 and IGR J00291+5934 show similar color variations during the outburst decay (Altamirano et al. 2010; Linares et al. 2007). The hardness-intensity diagram of IGR J17511-3057 is given in Fig. 2 of Altamirano et al. (2010), and shows a stable (hard) color up to $\sim 18$ days after the onset of the outburst, then the color softens within the hard state. Our Fig. 4 also shows that the product $\tau \times k T_{\mathrm{e}}$, as measured during the outburst of IGR J17511-3057 is stable during the outburst, in agreement with observations of other AMXPs (e.g., Gilfanov et al. 1998; Gierliński \& Poutanen 2005; Falanga et al. 2005a; Poutanen 2006; Ibragimov \& Poutanen 2009). This behavior is expected if the energy dissipation takes place in an accretion shock. The shock geometry can be approximated by a slab, and the cooling of the hot electron gas is determined by the reprocessing of the hard X-ray radiation at the neutron star surface (see Haardt \& Maraschi 1993; Stern et al. 1995; Poutanen \& Svensson 1996; Malzac et al. 2001). The temperature depends on the optical depth, but $\tau_{\mathrm{T}} \times k T_{\mathrm{e}}$ is approximately constant. In IGR J17511-3057 we observe $k T_{\mathrm{e}} \times \tau_{\mathrm{T}} \approx 50 \mathrm{keV}$ (see Fig. 4), which is consistent with the values determined for other 
AMXPs (Poutanen \& Gierliński 2003; Gierliński \& Poutanen 2005; Falanga et al. 2005a,b; Poutanen 2006) and also with the theoretical models.

The spectra of AMXPs are very similar to those of the atoll sources at low luminosities (Barret et al. 2000), where the X-rays are probably produced in the boundary/spreading layer near the NS equator (Kluźniak \& Wilson 1991; Inogamov \& Sunyaev 1999; Suleimanov \& Poutanen 2006). Spectral similarities can be explained if in both types of sources the energy dissipation happens in the optically thin medium (i.e. accretion shock and boundary/spreading layer) and the spectral properties are determined solely by energy balance and feedback from the NS surface which, provides cooling in the form of soft photons.

\section{Timing characteristics}

We also studied the pulsed emission of IGR J17511-3057 in the 3-300 keV energy range using RXTE/PCA, HEXTE, and INTEGRAL/ISGRI data. Because the flux decays over the course of the INTEGRAL observation we selected only those RXTE observations that overlap with the INTEGRAL observation lasting from MJD 55090.926 to 55093.520 (UTC) (see Fig. 2). In addition to the application of standard selection criteria in the screening process, the RXTE/PCA data were further screened for bursts and detector break-downs. The screening process yielded the following exposure times for PCU detectors 0-4, respectively: $16.096 \mathrm{ks}, 12.960 \mathrm{ks}, 47.344 \mathrm{ks}, 10.848 \mathrm{ks}$, and $13.216 \mathrm{ks}$. For a discussion of timing behavior over the whole outburst we refer the reader to Papitto et al. (2010a); Riggio et al. (2011); Ibragimov et al. (2010).

For HEXTE we only selected the onsource data streams from both detector clusters. The dead time-corrected exposure times in this case are 30.2 and $16.0 \mathrm{ks}$ for $\mathrm{HEXTE}^{2}$ Cluster 0 and 1 , respectively, for the period overlapping with the INTEGRAL observation. For the entire 94041 observation the effective exposure times were 232.4 and $123.2 \mathrm{ks}$ for Cluster 0 and 1 , respectively.

We screened the ISGRI data for effects caused by Earth radiation belt passages or solar flare activity, but none of the pointings showed such an activity. Time periods in which burst events occurred from any source in the ISGRI field of view were excluded from the subsequent analysis. Furthermore, we selected only time stamps of events with rise times between channels 7 and 90 (Lebrun et al. 2003), detected in non-noisy detector pixels, which have an illumination factor of more than $25 \%$.

The selected time stamps of all instruments were then converted to arrival times at the Solar System barycenter, taking into account the orbital motion of the spacecraft and correcting for acceleration effects along the binary orbit. In this process we used the position of the optical counterpart to IGR J17511-3057 as reported by Torres et al. (2009a,b).

\subsection{Pulse profiles and time lags}

Pulse phase folding of the barycented arrival times using the ephemeris given in Papitto et al. (2009) yielded the pulsephase distributions shown in Fig. 5. We added for the PCA data (collected in mode E_125us_64M_0_1s) a time shift of $+0.5 \times 122.07 \mu$ s to the barycented time stamps because the times referred to the start of the time bin instead of the mid of the bin. Panels a-d of Fig. 5 show the PCA pulse profiles for the energy

\footnotetext{
$\overline{2}$ HEXTE Cluster 0 operates in staring mode since July 13, 2006.
}

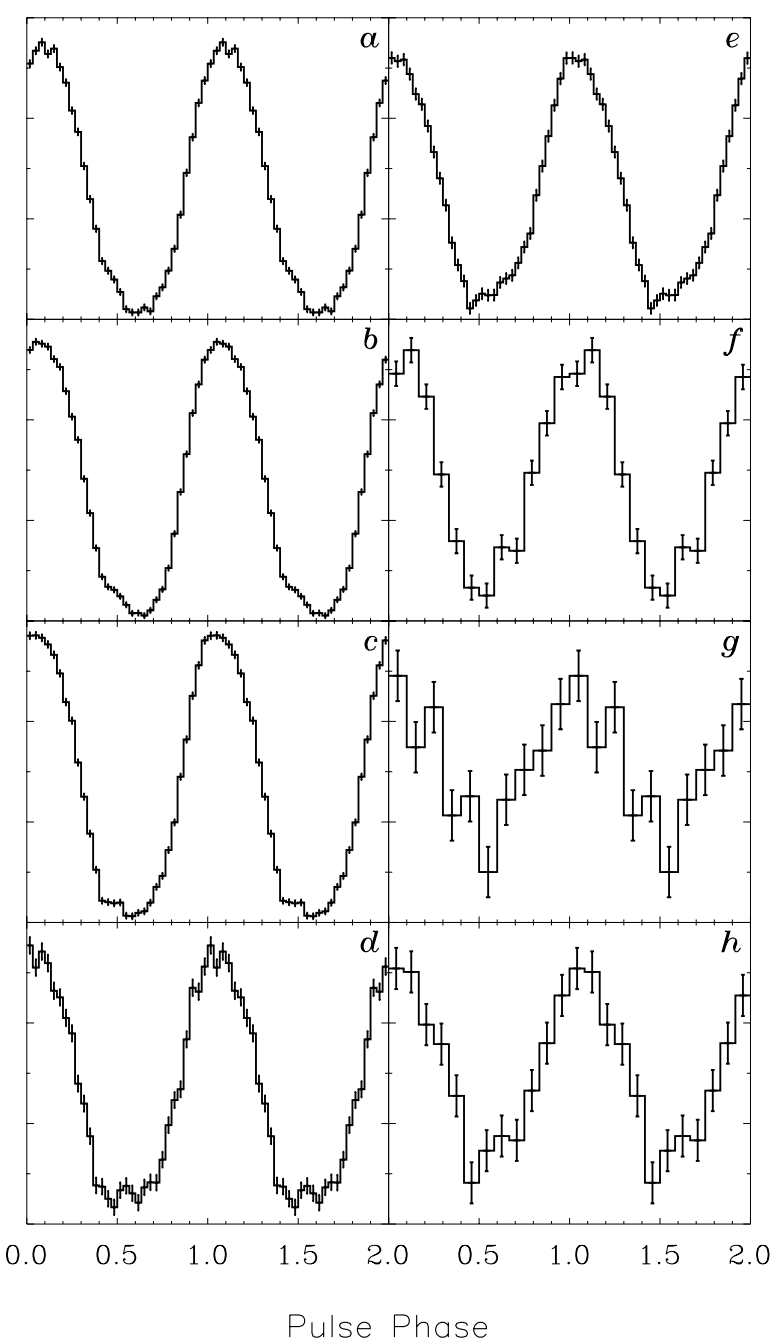

Fig. 5. Pulse-profile collage of IGR J17511-3057 using data from RXTE/PCA, HEXTE, and INTEGRAL/ISGRI. Panels a-d) (PCA) correspond to the energy intervals $1.7-4.2,4.2-7.9,7.9-16.2$ and $16.2-$ $34.9 \mathrm{keV}$. Panels $\mathbf{e}-\mathbf{g}$ ) show the HEXTE pulse profiles in the bands 15.6-31.0, 31.0-60.1 and 60.1-123.9 keV (accumulated during the entire 94041 observation period) in panel h) the ISGRI profile $15-90 \mathrm{keV}$. The error bars represent $1 \sigma$ statistical errors. All profiles reach their maximum near phase $\sim 0.1$. The $y$-axis is given in units of counts per bin.

intervals, 1.7-4.2, 4.2-7.9, 7.9-16.2 and 16.2-34.9 keV, respectively. In panels e-g the HEXTE profiles (accumulated during the entier 94041 observation) are shown for the bands 15.6-31.0, 31.0-60.1 and 60.1-123.9 keV, and panel h shows the ISGRI profile for energies between 15 and $90 \mathrm{keV}$. Pulsed emission was detected up to $\sim 120 \mathrm{keV}$ using HEXTE data collected during the full duration of observation 94041. The mutual alignment of the RXTE/PCA, HEXTE, and INTEGRAL/ISGRI profiles within the equivalent energy bands is better than $50 \mu$ s. The profiles are fairly sinusoidal with the amplitudes of harmonics not exceeding $15 \%$ of the fundamental (see Papitto et al. 2010a). A small asymmetry could be caused by a strong deviation of emission pattern from the blackbody (Poutanen \& Gierliński 2003; Viironen \& Poutanen 2004) and/or the appearance at some phases of the secondary spot (Papitto et al. 2010a) as seen in SAX J1808.4-3658 (Ibragimov \& Poutanen 2009; Poutanen et al. 2009).

We studied the global arrival times of the pulses as a function of energy and used the PCA profile for channel 1 


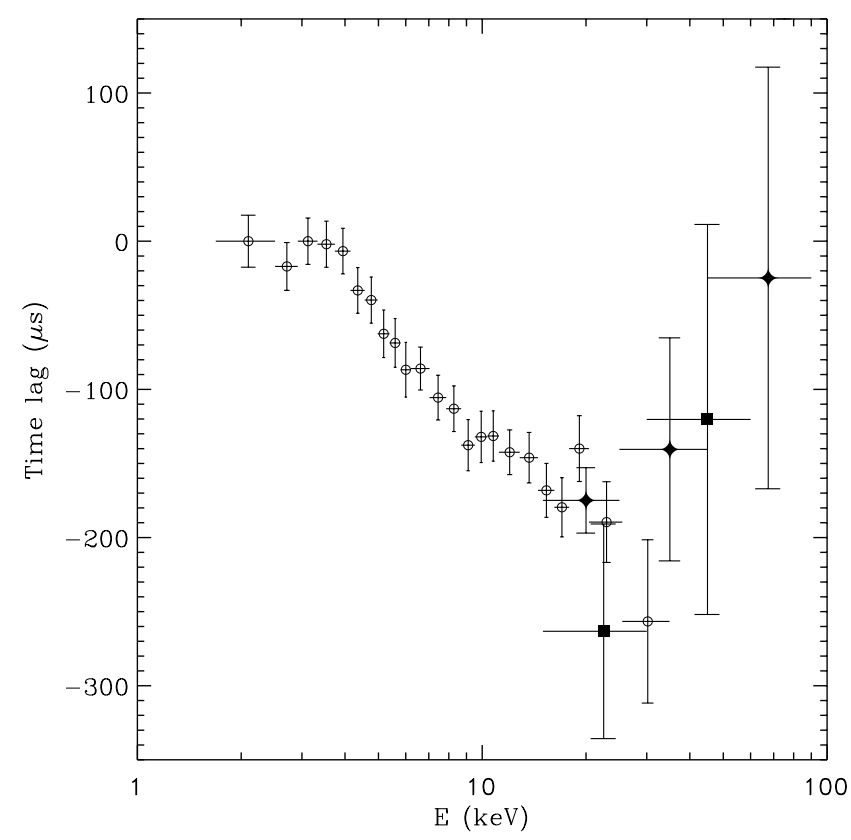

Fig. 6. Time lag as a function of energy in the $\sim 2-100 \mathrm{keV}$ energy range combining RXTE/PCA (1.7-35 keV; open circles), RXTE/HEXTE (15-90 keV; filled diamonds), and INTEGRAL/ISGRI (15-90 keV; filled squares) measurements.

$(\sim 1.69-2.51 \mathrm{keV})$ as a reference template. Cross-correlation of the pulse profiles obtained for other energy bands with this template yielded the time lags shown in Fig. 6 . Beyond $\sim 4 \mathrm{keV}$ a declining trend sets in, meaning that the hard X-ray photons arrive earlier than the soft ones.

The behavior of the IGR J17511-3057 time lags is similar to that observed in XTE J1751-305. In these cases the low-energy pulses lag behind the high-energy pulses (soft phase/time lags), which monotonically increase with energy and saturate at about 10-20 keV (Gierliński \& Poutanen 2005). The saturation energy is, however, much lower in IGR J00291+5934 (see e.g. Falanga et al. 2005b; Falanga \& Titarchuk 2007) and SAX J1808.4-3658 (Cui et al. 1998; Poutanen \& Gierliński 2003; Poutanen 2006; Ibragimov \& Poutanen 2009), where the time lag as a function of energy breaks at about $7 \mathrm{keV}$. For IGR J00291+5934 the soft lag even decreases beyond this value.

The lags are most probably caused by different emission patterns of the blackbody and Comptonization components (Poutanen \& Gierliński 2003; Gierliński \& Poutanen 2005; Ibragimov \& Poutanen 2009) combined with the action of the Doppler effect. This is supported by the energy dependence of the lags, which grows until the contribution of the blackbody becomes negligible. At higher energies, the evolution of the soft lag is still further possible, because higher-energy photons suffer more scatterings resulting in variations of the emission pattern with energy (Poutanen \& Gierliński 2003; Viironen \& Poutanen 2004). The break in the lag spectrum at a higher energy might be a result of a higher seed blackbody temperature for Comptonization, which in turn implies a smaller spot area, and/or a smaller contribution of the blackbody to the total spectrum. Note that in XTE J1751-305 (Gierliński \& Poutanen 2005) and IGR J17511-3057 the blackbody is hardly visible, while it is very apparent in the spectra of IGR J00291+5934 (Falanga et al. 2005b), and SAX J1808.4-3658 (Gierliński et al. 2002; Poutanen \& Gierliński 2003; Poutanen 2008). A smaller blackbody area indicates either a larger magnetic field in the first two sources compared to IGR J00291+5934, or a different geometry (e.g. inclination), or a different optical depth of the Comptonizing plasma in the accretion shock.

\subsection{Spectrum of the pulsed emission and pulsed fraction}

The pulsed spectrum and pulsed fraction (defined as pulsed flux/total flux) as a function of energy provide important diagnostic parameters for constraining the parameter space in theoretical modeling (see e.g. Viironen \& Poutanen 2004). We derived the pulsed fluxes $(\sim 3-120 \mathrm{keV})$ from observations with the non-imaging RXTE/ PCA and HEXTE instruments overlapping in time with the INTEGRAL observation, and the (imaging) ISGRI instrument. The total fluxes $(\sim 0.8-300 \mathrm{keV})$ have been determined from instruments with imaging capabilities, namely Swift/XRT, and JEM-X and ISGRI aboard INTEGRAL (see also Sect. 4.1).

Firstly, we derived the pulsed excess counts (=counts above DC level) in a given energy band by fitting a truncated Fourier series, using only the fundamental and two harmonics, to the measured pulse phase distribution. For the PCA data ( 3$35 \mathrm{keV}$ band) the pulsed excess counts were converted to photon flux values ( $\mathrm{ph} \mathrm{cm}^{-2} \mathrm{~s}^{-1} \mathrm{keV}^{-1}$ ) in a forward spectral folding procedure assuming an underlying power-law model taking into account the different exposure times (see Sect. 5) and energy responses of the five active PCU's. We kept the absorbing hydrogen column density fixed to $6.0 \times 10^{21} \mathrm{~cm}^{-2}$ (see Table 1), although its precise value has very little impact on the fit results because we consider only measurements with energies above $3 \mathrm{keV}$. We obtained an unabsorbed 2-10 keV (pulsed) flux of $8.09(6) \times 10^{-11} \mathrm{erg} \mathrm{cm}^{-2} \mathrm{~s}^{-1}$ and a power-law index of $1.824 \pm 0.004$ (reduced $\chi^{2}$ of the fit was $14.34 /(21-2)$ ), indicating that the pulsed spectrum is softer than the total spectrum (see Table 1).

For the HEXTE data $(\sim 15-120 \mathrm{keV})$ we employed an equivalent method: the pulsed excess counts in a certain energy band (for those RXTE observations overlapping with the INTEGRAL observation) were divided by its effective sensitive area assuming a power-law model with index 1.824 , taking into account the different energy responses and dead-time corrected exposure times of the two detector clusters. Finally, the pulsed ISGRI excess counts ( $\sim 15-90 \mathrm{keV})$ of IGR J17511-3057 have been converted to flux values adopting the method outlined in Sect. 3.4 of Kuiper et al. (2006).

The (unabsorbed) PCA, HEXTE and ISGRI pulsed flux measurements are shown in Fig. 7 along with the (unabsorbed) total flux measurements. If we compare the energy spectrum of the pulsed emission of IGR J17511-3057 and IGR J00291+5934 (see Falanga et al. 2005a), then we see a significant difference: in the $E F_{E}$ spectral representation the pulsed flux of IGR J17511-3057 shows a cut-off near $20 \mathrm{keV}$, while that of IGR J00291+5934 increases up to the end of the sensitivity window. From the pulsed and total flux measurements shown in Fig. 7 we can directly derive the pulsed fraction as a function of energy which is shown in Fig. 8. The pulsed fraction decreases from $\sim 22 \%$ at $3 \mathrm{keV}$ to a constant pulsed fraction of $\sim 17-18 \%$ between $7-30 \mathrm{keV}$, and then decreases again until $\sim 13 \%$ at $60 \mathrm{keV}$. The decreasing part is very similar to what is observed in XTE J1751-305 (Gierliński \& Poutanen 2005), while in IGR J00291+5934 the pulsed fraction has a minimum of about $\sim 6 \%$ at $7 \mathrm{keV}$ and grows again to $\sim 12-20 \%$ at $100 \mathrm{keV}$ (Falanga et al. 2005a). 
Table 2. Burst characteristics observed with RXTE/PCA and INTEGRAL/JEM-X.

\begin{tabular}{lccccccc}
\hline \hline$\#$ & $\begin{array}{c}\text { Burst } T_{\text {start }} \\
(\mathrm{UT})\end{array}$ & $\begin{array}{c}F_{\text {pers,bol }}{ }^{a} \\
10^{-9} \mathrm{erg} \mathrm{cm}^{-2} \mathrm{~s}^{-1}\end{array}$ & $\begin{array}{c}F_{\text {peak }}{ }^{b} \\
10^{-9} \mathrm{erg} \mathrm{cm}^{-2} \mathrm{~s}^{-1}\end{array}$ & $\begin{array}{c}f_{\mathrm{b}}{ }^{c} \\
10^{-7} \mathrm{erg} \mathrm{cm}^{-2}\end{array}$ & $\begin{array}{c}\tau_{\mathrm{b}}{ }^{d} \\
\mathrm{~s}\end{array}$ & $\begin{array}{c}k T_{\text {bb,peak }}{ }^{e} \\
\mathrm{keV}\end{array}$ & $\begin{array}{c}R_{\text {bb,peak }}{ }^{f} \\
\mathrm{~km}\end{array}$ \\
\hline 2 & $2009-09-1407: 54: 43$ & $1.63 \pm 0.2$ & $34.9 \pm 1.2$ & $3.20 \pm 0.03$ & $9.2 \pm 0.3$ & $2.69 \pm 0.06$ & $5.5 \pm 0.3$ \\
3 & $2009-09-1517: 17: 23$ & $1.17 \pm 0.2$ & $33.0 \pm 1.2$ & $3.48 \pm 0.04$ & $10.5 \pm 0.4$ & $2.59 \pm 0.05$ & $5.8 \pm 0.3$ \\
4 & $2009-09-1706: 33: 27$ & $1.48 \pm 0.2$ & $38.5 \pm 1.2$ & $3.74 \pm 0.04$ & $9.0 \pm 0.3$ & $2.93 \pm 0.06$ & $4.8 \pm 0.2$ \\
5 & $2009-09-1714: 48: 42$ & $1.49 \pm 0.2$ & $41.6 \pm 1.2$ & $3.52 \pm 0.04$ & $8.5 \pm 0.3$ & $2.99 \pm 0.06$ & $4.9 \pm 0.2$ \\
$6^{g}$ & $2009-09-1808: 09: 29$ & $1.28 \pm 0.2$ & $45.0 \pm 9.0$ & $3.30 \pm 0.6$ & $6.6 \pm 1.9$ & $3.60 \pm 0.9$ & $4.74 \pm 1.8$ \\
$7^{9}$ & $2009-09-1902: 28: 20$ & $1.31 \pm 0.2$ & $38.0 \pm 9.0$ & $3.40 \pm 0.7$ & $7.4 \pm 2.5$ & $3.40 \pm 0.9$ & $4.74 \pm 1.8$ \\
$8^{9}$ & $2009-09-1911: 13: 03$ & $1.38 \pm 0.2$ & $36.6 \pm 10$ & $3.90 \pm 0.7$ & $7.9 \pm 2.9$ & $3.20 \pm 0.6$ & $4.8 \pm 1.8$ \\
9 & $2009-09-2014: 50: 31$ & $1.36 \pm 0.2$ & $42.4 \pm 1.2$ & $3.67 \pm 0.03$ & $8.6 \pm 0.3$ & $2.92 \pm 0.07$ & $5.1 \pm 0.3$ \\
13 & $2009-09-2314: 27: 06$ & $1.01 \pm 0.2$ & $48.5 \pm 1.2$ & $3.89 \pm 0.04$ & $8.0 \pm 0.2$ & $3.26 \pm 0.1$ & $4.4 \pm 0.3$ \\
14 & $2009-09-2418.39: 13$ & $0.95 \pm 0.2$ & $48.4 \pm 1.2$ & $3.84 \pm 0.04$ & $7.9 \pm 0.2$ & $3.15 \pm 0.09$ & $5.4 \pm 0.3$ \\
15 & $2009-09-2507: 31: 36$ & $1.02 \pm 0.2$ & $53.2 \pm 1.2$ & $3.94 \pm 0.02$ & $7.4 \pm 0.2$ & $3.09 \pm 0.06$ & $5.2 \pm 0.2$ \\
16 & $2009-09-2615: 11: 22$ & $0.94 \pm 0.2$ & $53.7 \pm 1.2$ & $4.18 \pm 0.05$ & $7.8 \pm 0.2$ & $2.98 \pm 0.07$ & $5.6 \pm 0.3$ \\
17 & $2009-09-2706: 57: 21$ & $0.88 \pm 0.2$ & $56.9 \pm 1.2$ & $4.21 \pm 0.04$ & $7.4 \pm 0.2$ & $3.15 \pm 0.09$ & $5.1 \pm 0.3$ \\
\hline
\end{tabular}

Notes. ${ }^{(a)}$ Pre-burst unabsorbed flux in $0.8-200 \mathrm{keV}$ energy range. ${ }^{(b)}$ Burst peak flux in $0.1-40 \mathrm{keV}$ energy band. ${ }^{(c)}$ Burst fluence in $0.1-40 \mathrm{keV}$ energy band. ${ }^{(d)}$ Effective duration $\tau_{\mathrm{b}}=f_{\mathrm{b}} / F_{\text {peak }} .{ }^{(e)}$ Burst peak temperature. ${ }^{(f)}$ Burst peak blackbody radius for a distance of $7 \mathrm{kpc} .{ }^{(g)}$ Bursts detected with INTEGRAL/JEM-X.

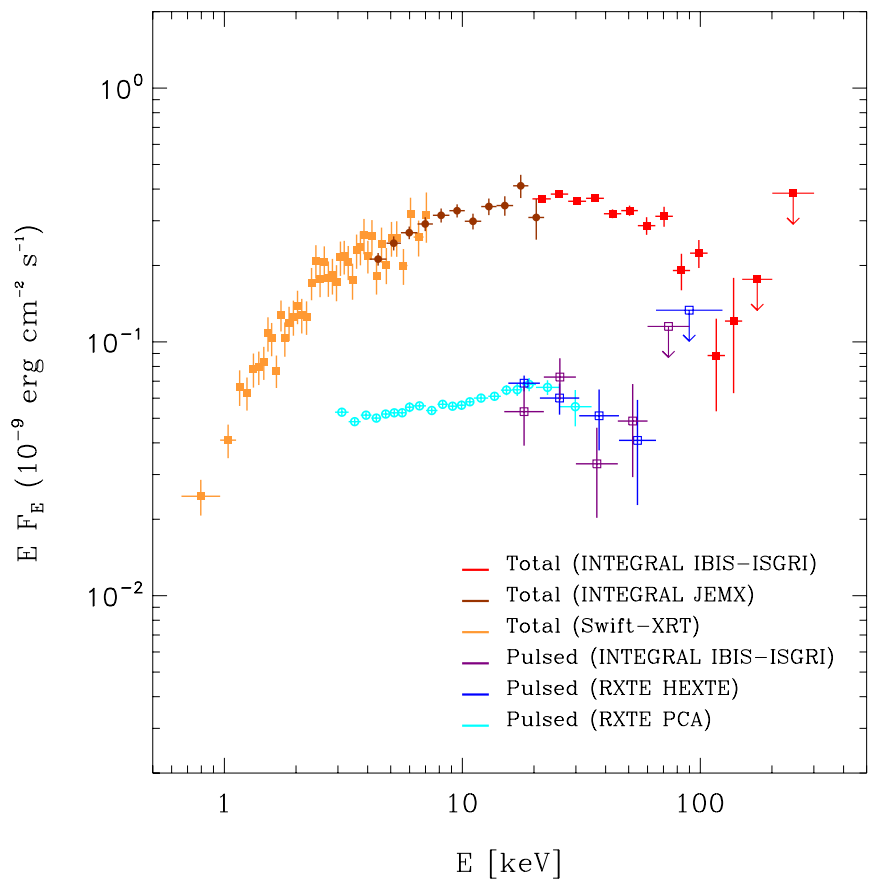

Fig. 7. Unabsorbed total $(\sim 0.8-300 \mathrm{keV})$ and pulsed $(\sim 3-120 \mathrm{keV})$ unfolded spectra of IGR J17511-3057 combining measurements from RXTE/PCA, HEXTE, and INTEGRAL/ISGRI for the pulsed part and Swift/XRT, INTEGRAL/JEM-X and ISGRI for the total part.

\section{Properties of the X-ray bursts}

In Table 2 we report the key measurable parameters for the bursts observed from IGR J17511-3057. Thermonuclear (type-I) X-ray bursts are produced by unstable burning of accreted matter on the NS surface. The spectrum from a few keV to higher energies can usually be well described as a blackbody with temperature $k T_{\mathrm{bb}} \approx 1-3 \mathrm{keV}$. The energy-dependent decay time of these bursts is attributed to the cooling of the NS photosphere and results in a gradual softening of the burst spectrum (see Lewin et al. 1993; Strohmayer \& Bildsten 2006, for a review).

We defined the burst start time as the time at which the X-ray intensity of the source first exceeded $10 \%$ of the burst peak flux (above the persistent intensity level). The time-resolved spectral

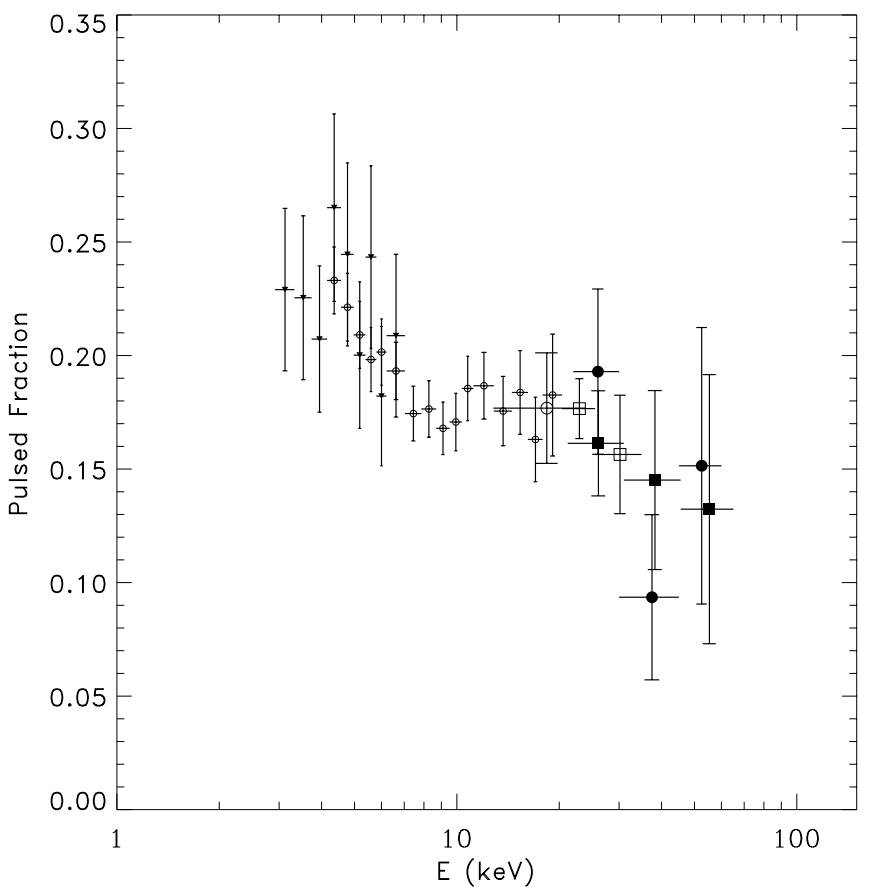

Fig. 8. The pulsed fraction (=pulsed flux/total flux) of IGR J175113057 based on the pulsed/total flux measurements from Swift/XRT, RXTE/PCA/HEXTE, and INTEGRAL/JEM-X/ISGRI.

analysis of the 13 bursts (10 RXTE and 3 INTEGRAL) was carried out by using RXTE/PCA and INTEGRAL/JEM-X data in the $2.5-20 \mathrm{keV}$ and 3-20 keV bands, respectively. From these analyses we determined the bursts' peak fluxes, temperatures, and radii (see Table 2). We fitted each burst spectra by a simple photoelectrically-absorbed blackbody model, вв. The neutral absorption column density $N_{\mathrm{H}}$ was left free to vary in the range (2.6-0.001) $10^{22} \mathrm{~cm}^{-2}$ in all fits. However, we checked that fixing $N_{\mathrm{H}}=0.6 \times 10^{22} \mathrm{~cm}^{-2}$ did not significantly affect the results. We extrapolated the unabsorbed fluxes to the $0.2-50 \mathrm{keV}$ band by generating dummy responses (XSPEC version 12.6). This is justifiable for the data because the blackbody temperature is well inside the spectral bandpass. The inferred вв peak temperature, $k T_{\text {bb,peak }}$, apparent вв radius at $7 \mathrm{kpc}$ (see below), $R_{\text {bb,peak }}$, and 


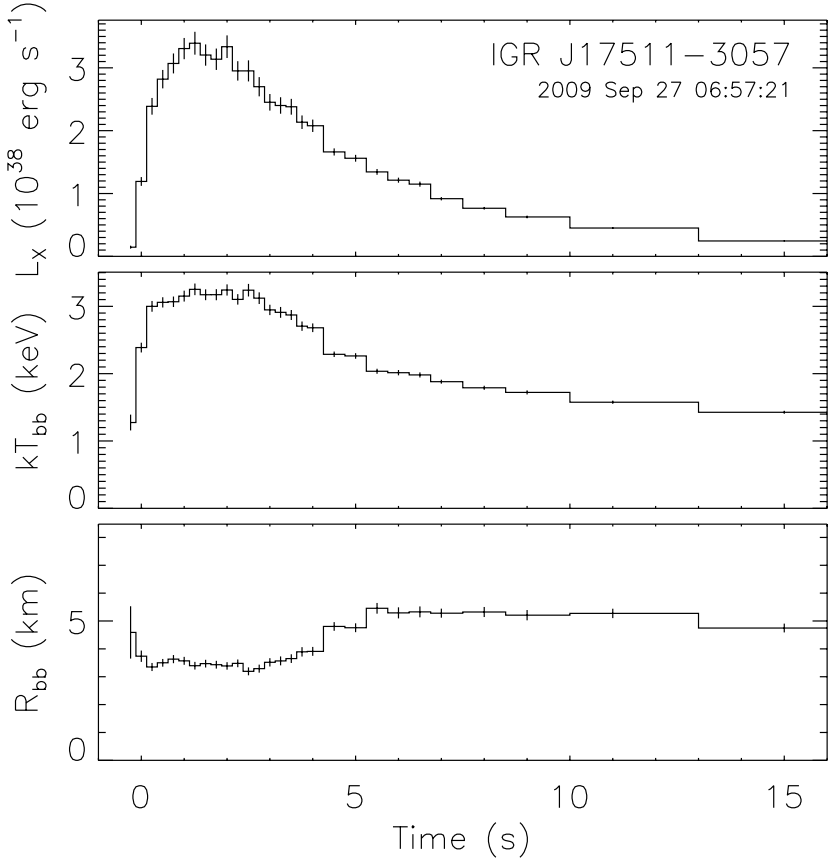

Fig. 9. Representative time-resolved spectroscopic results from the brightest burst seen by RXTE from IGR J17511-3057. We show the inferred bolometric luminosity from integrating the best-fit blackbody model (top panel); the blackbody temperature (middle panel); and the effective blackbody radius (bottom panel), assuming a distance of $7 \mathrm{kpc}$.

unabsorbed bolometric peak flux are also reported in Table 2. In Fig. 9 we show representative time-resolved spectroscopic parameters.

We calculated the burst fluence, $f_{\mathrm{b}}$, by integrating the flux over the burst duration. The effective burst duration is $\tau_{\mathrm{b}}=$ $f_{\mathrm{b}} / F_{\text {peak }}$. All bursts are short, with $\tau_{\mathrm{b}}$ in the range $7-10 \mathrm{~s}$. The fluence and peak flux for the bursts steadily increased with time, from $\sim 3.0 \times 10^{-7}$ to $4.2 \times 10^{-7} \mathrm{erg} \mathrm{cm}^{-2} \mathrm{~s}^{-1}$ for the fluence, and from $\sim 3.3 \times 10^{-9}$ to $5.7 \times 10^{-9} \mathrm{erg} \mathrm{cm}^{-2} \mathrm{~s}^{-1}$ for the peak flux. As a consequence, $\tau_{b}$ steadily decreased, and the rise time also decreased, from $\sim 2$ to $\sim 1 \mathrm{~s}$. The brightest burst reached

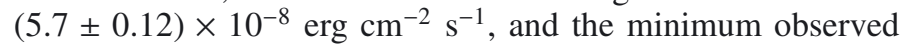
separation of the bursts was $7.08 \mathrm{~h}$ (see Table 3 ). These results agree with these reported in Altamirano et al. (2010). Those authors also reported the properties of burst oscillations detected at the pulse frequency in all bursts.

When a burst undergoes a photospheric-radius expansion (PRE), the source distance can be determined based on the assumption that the bolometric peak luminosity is saturated at the Eddington limit, $L_{\text {Edd }}$ (e.g., Lewin et al. 1993; Kuulkers et al. 2003). None of the observed bursts exhibited PRE; thus, in these cases the peak luminosity is expected to have been sub-Eddington, which leads to an upper limit on the distance. Assuming a bolometric peak luminosity equal to the Eddington value for a He X-ray burst $\left(L_{\mathrm{Edd}} \approx 3.8 \times 10^{38} \mathrm{ergs}^{-1}\right.$, as empirically derived by Kuulkers et al. 2003), we obtain using the brightest burst (see Table 2) the source distance upper-limit of $d \lesssim 7.5 \mathrm{kpc}$. For comparison, the theoretical value of this upper limit distance (e.g., Lewin et al. 1993) found by assuming a $\mathrm{He}$ atmosphere and canonical NS parameters (1.4 solar mass and radius of $10 \mathrm{~km}$ ), is $\sim 6.3 \mathrm{kpc}$. Alternatively, assuming the peak luminosity to be the Eddington luminosity for solar composition $(X=0.7)$ implies a limit of $\approx 4.8 \mathrm{kpc}$. Below, we consider $d \approx 7 \mathrm{kpc}$ to be a fiducial distance. At this distance, all bursts occurred at persistent luminosities between $(5.2-9.6) \times 10^{36} \mathrm{erg} \mathrm{s}^{-1}$ (see Table 2 ), or $\approx(1.4-2.5) \% L_{\text {Edd }}$ using $L_{\text {Edd }} \approx 3.8 \times 10^{38} \mathrm{erg} \mathrm{s}^{-1}$. The local accretion rate per unit area for the pre-burst emission, $L_{\text {pers }}$, is then given by $\dot{m}=$ $L_{\text {pers }}(1+z)\left(4 \pi R^{2}(G M / R)\right)^{-1}$, i.e. $\dot{m} \sim(2.13-5.3) \times 10^{3} \mathrm{~g} \mathrm{~cm}^{-2} \mathrm{~s}^{-1}$. We use here the gravitational redshift $1+z=1.31$ for the canonical NS mass, $M=1.4 M_{\odot}$, and radius, $R=10 \mathrm{~km}$.

The observed energies of the bursts allow us to estimate the ignition depths. The measured fluences of the bursts are $f_{\mathrm{b}}=(3.2-4.2) \times 10^{-7} \mathrm{erg} \mathrm{cm}{ }^{-2}$, corresponding to a net burst energy release $E_{\text {burst }}=4 \pi d^{2} f_{\mathrm{b}}=(1.9-2.5) \times 10^{39}(d / 7 \mathrm{kpc})^{2} \mathrm{erg}$. The ignition depth is given by $y_{\text {ign }}=E_{\text {burst }}(1+z)\left(4 \pi R^{2} Q_{\text {nuc }}\right)^{-1}$, where the nuclear energy generated (assuming a mean hydrogen mass fraction at ignition $\langle X\rangle)$ is $Q_{\mathrm{nuc}} \approx 1.6+4\langle X\rangle \mathrm{MeV} /$ nucleon (Galloway et al. 2004, and references therein), including losses owing to neutrino emission following Fujimoto et al. (1987).

Most burst sources accrete a mix of hydrogen and helium, although the $\mathrm{H}$-fraction at ignition may also be reduced by steady burning between the bursts, i.e. $X \leq X_{0}$. Steady H-burning proceeds via the hot-CNO cycle, which will exhaust the available hydrogen in approximately $11 \mathrm{~h}(Z / 0.02)^{-1}\left(X_{0} / 0.7\right)$, where $Z$ is the mass fraction of CNO nuclei, and $X_{0}$ the mass fraction of hydrogen in the accreted fuel (e.g., Galloway et al. 2004). As for all bursts observed from IGR J17511-3057, the bursts early in the outburst, when the recurrence time was as short as $7 \mathrm{~h}$, exhibit short profiles, characteristic of low $\mathrm{H}$-fraction, and with correspondingly high $\alpha$-values. In order to exhaust the accreted $\mathrm{H}$ in just $7 \mathrm{~h}$ via steady burning, the accreted $\mathrm{H}$-fraction must be substantially below solar; we infer $X_{0} \leq 0.44(Z / 0.02)$. Naturally, it is also possible that the H-fraction is closer to the solar value, or that the CNO metallicity is elevated. For the inferred pure helium composition at ignition (i.e. $\langle X\rangle=0$ ) the column depth varies little from burst to burst, in the range $y_{\text {ign }}=(1.2-1.6) \times 10^{8} \mathrm{~g} \mathrm{~cm}^{-2}$.

Once steady H-burning exhausts the accreted hydrogen at the base of the fuel layer, this process no longer dominates heating in the layer, and ignition will occur in a pure He-layer (case 2 of Fujimoto et al. 1981). As the accretion rate decreases through the decay of the outburst, we expect the burst recurrence times to become longer, as is observed. We found that the recurrence time increases roughly as $\left\langle F_{\text {pers,bol }}\right\rangle^{-1.1}$ (Fig. 10), where $\left\langle F_{\text {pers, bol }}\right\rangle$ is the averaged persistent flux between the bursts. However, the expected increase in the burst recurrence time in the pure $\mathrm{He}$ ignition regime as a function of decreasing $\dot{m}$ is much steeper. For example, the curve for $X_{0}=0.3$ in Fig. 1 of Galloway \& Cumming (2006) falls off roughly as $\dot{m}^{-3.5}$, substantially steeper than measured in IGR J17511-3057. What is also puzzling is that the measured $\alpha$-value (the ratio of persistent to burst luminosity) decreases with decreasing $\left\langle F_{\text {pers,bol }}\right\rangle$. As the burst recurrence time increases, the fuel layer should become increasingly He-rich, so that a slight increase in $\alpha$ would be expected.

This analysis relies on unambiguous measurement of the burst recurrence time, for which we used all bursts reported in the literature, with Swift (Bozzo et al. 2009), XMM-Newton (Papitto et al. 2010a), and Chandra (Nowak et al. 2009) in addition the INTEGRAL and RXTE. In some cases, the effective exposure time onsource from burst to burst was shorter then the expected recurrence time between bursts, so that we most likely missed one or more bursts between the observed one (see Table 3). Therefore, we divided some measured times between bursts by an integer number, $\Delta t_{\text {rec }} /(N+1)$ (see Table 3 ), where $N$ is the number of missed bursts. We verified that at the expected burst times we always had an observational data gap. With the inclusion of observations for which there were no intervening 
Table 3. Burst recurrence times and effective exposure times.

\begin{tabular}{|c|c|c|c|c|c|}
\hline \# & Observatory & $T_{\text {start }}$ & $\Delta t_{\text {rec }}(\mathrm{h})$ & $T_{\exp }(\mathrm{h})$ & $N$ \\
\hline 1 & Swift & $2009-09-14$ 00:50:27 & - & - & - \\
\hline 2 & & & & 1.515 & - \\
\hline 3 & E/Swift & & & & 3 \\
\hline 4 & XTE & 200 & & & 3 \\
\hline 5 & $\mathrm{E}$ & 200 & & 549 & - \\
\hline 6 & INTEG & 2009 & 31 & 859 & 1 \\
\hline 7 & & 200 & & & 1 \\
\hline 8 & & 200 & & 226 & - \\
\hline 9 & RXTE & 2005 & & 5.056 & 2 \\
\hline 10 & $X M M-N$ & 200 & & 2.40 & - \\
\hline 11 & $X M M-$ & 2009 & & 361 & - \\
\hline 12 & Chandra & 2009 & & 12.555 & 1 \\
\hline 13 & RXTE & 2009 & & 207 & 1 \\
\hline 14 & RXTE & 2009 & 28.214 & 10.976 & 1 \\
\hline 15 & & $200 c$ & & 6.133 & - \\
\hline 16 & & & & & \\
\hline 17 & RXTE & 2009-09-27 & & 4.985 & - \\
\hline 18 & Swift & 2009-09-30 18:31:57 & 83.576 & 24.240 & \\
\hline
\end{tabular}

Notes. Measured recurrence time between observed bursts is $\Delta t_{\mathrm{rec}}$. The effective exposure time, $T_{\text {exp }}$, takes into account all the instruments observations. In Fig. 10 the triangles corresponds to $\Delta t_{\text {rec }} /(N+1)$, with $N$ being the number of the expected missed bursts.

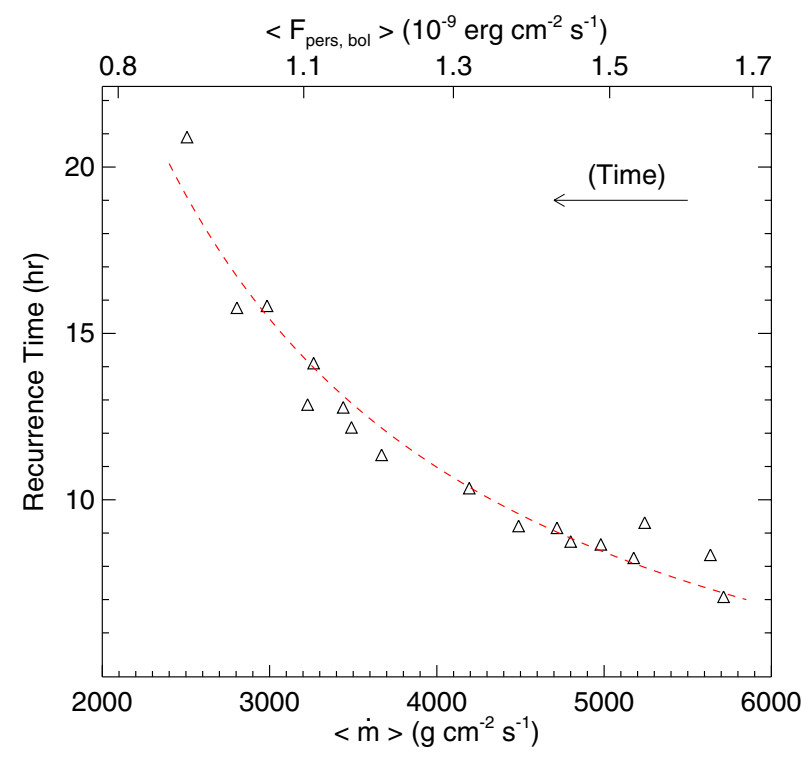

Fig. 10. Triangles in the figure represent the observed burst recurrence times (see Table 3) shown as a function of local mass accretion rate (the corresponding flux is reported in the upper $x$-axis). We also show the best-fit power-law model. The recurrence time increased with time roughly as $\left\langle F_{\text {pers,bol }}\right\rangle^{-1.1}$.

data gaps (e.g. with XMM-Newton), we are confident that we have precisely inferred the recurrence time between each pair of bursts listed in Table 3.

The discrepancy between the expected and observed variation in recurrence time is substantial, and in contrast to the (otherwise fairly similar) bursts observed at comparable accretion rates in SAX J1808.4-3658 (Galloway \& Cumming 2006). As is often argued, it is possible that the variation in $\dot{m}$ is not as large as is suggested by the decrease in $F_{\text {pers,bol }}$. If, as the X-ray intensity drops, we are seeing a decreasing fraction of the accretion energy in the X-ray band, the actual $\dot{m}$ could remain higher, which would be consistent with both the moderate recurrence times and the decreasing $\alpha$ values. However, this would require a substantial change in the efficiency of the $\dot{m}$ to $F_{\text {pers,bol }}$ conversion. One way this could occur is that if the spectral energy distribution changes in a way that the accretion flux is increasingly emitted outside the band we are sensitive to. However, the combination of instruments with both low- and high-energy sensitivity (Swift \& INTEGRAL) makes this unlikely. Thompson et al. (2008) found from a detailed study of GS 1826-24 that the characteristic uncertainty that might arise from this mechanism is $40 \%$. This is not sufficient to explain the discrepancy in IGR J17511-3057.

\section{Summary}

We analyzed the simultaneous INTEGRAL, RXTE, and Swift observations to study the broad-band spectrum and timing behavior of IGR J17511-3057. Using all RXTE data we also studied the outburst profile. The broad-band average spectrum is well described by thermal Comptonization with an electron temperature of $\sim 25 \mathrm{keV}$ and Thomson optical depth $\tau_{\mathrm{T}} \sim 2$ in a slab geometry. The object shows remarkable spectral stability during the outburst, marked by constant plasma and seed photon temperature at a constant scattering optical depth. We fitted the outburst profile with the exponential model and using the disk instability model we inferred the outer disk radius to be $(4.8-5.4) \times 10^{10} \mathrm{~cm}$.

We showed that the coherent pulsation can be tracked with the HEXTE and ISGRI instruments up to $\sim 100 \mathrm{keV}$. The pulsed fraction is shown to decrease from $\sim 22 \%$ at $3 \mathrm{keV}$ to a constant of $\sim 17-18 \%$ between $7-30 \mathrm{keV}$, and then to possibly decrease even to $\sim 13 \%$ at $60 \mathrm{keV}$. This is similar to what is observed in XTE J1751-305, but markedly different from IGR J00291+5934. The nearly sinusoidal pulses show soft lags that monotonically increase with energy to about $0.2 \mathrm{~ms}$ at 10 $20 \mathrm{keV}$, with some indications of a further decrease.

Using all observations by INTEGRAL, RXTE, Swift, Chandra, and XMM-Newton we collected the most compresive set of X-ray bursts observed from IGR J17511-3057, which allowed us to determine the recurrence time (accounting for the missed bursts) as a function of the accretion rate and the ignition depth. The short burst profiles indicate hydrogen-poor material at ignition, which suggests either that the accreted material is hydrogen-deficient, or that the CNO metallicity is up to a factor of 2 times solar. However, the variation of the burst recurrence time as a function of $\dot{m}$ (inferred from the X-ray flux) is much smaller than predicted by helium-ignition models.

Acknowledgements. M.F. thanks C. Winkler and the INTEGRAL staff for the rapid schedule of the INTEGRAL observation of IGR J17511-3057 shortly after the onset of its outburst. J.P. acknowledges financial support from the Academy of Finland grant 127512.

\section{References}

Alpar, M. A., Cheng, A. F., Ruderman, M. A., \& Shaham, J. 1982, Nature, 300, 728

Altamirano, D., Watts, A., Linares, M., et al. 2010, MNRAS, 409, 1136

Arnaud, K. A. 1996, in Astronomical Data Analysis Software and Systems V, ed. G. H. Jacoby, \& J. Barnes, ASP Conf. Ser., 101 (San Francisco: ASP), 17 Baldovin, C., Kuulkers, E., Ferrigno, C., et al. 2009, ATel, 2196

Barret, D., Olive, J. F., Boirin, L., et al. 2000, ApJ, 533, 329

Bozzo, E., Ferrigno, C., Kuulkers E., et al. 2009, ATel, 2198

Bozzo, E., Ferrigno, C., Falanga, M., et al. 2010, A\&A, 509, L3 
Burrows, D. N., Hill, J. E., Nousek, J. E., et al. 2005, SSRv, 120, 165 Chakrabarty, D., Morgan, E. H., Muno, M. P., et al. 2003, Nature, 424, 42 Chenevez, J., Kuulkers, E., Beckmann, V., et al. 2009, ATel, 2235

Courvoisier, T. J.-L., Walter, R., Beckmann, V., et al. 2003, A\&A, 411, L57

Cui, W., Morgan, E., \& Titarchuk, L. 1998, ApJ, 504, 27

Deloye, C. J., \& Bildsten, L. 2003, ApJ, 598, 1217

Dickey, J. M., \& Lockman, F. J. 1990, ARA\&A, 28, 215

Di Salvo, T., \& Burderi, L. 2003, A\&A, 397, 723

Falanga, M. 2008, in Cool Discs, Hot Flows: The Varying Faces of Accreting Compact Objects. ed. M. Axelsson (Melville, NY: AIP), AIP Conf. Ser., 1054, 157

Falanga, M., \& Titarchuk, L. 2007, ApJ, 661, 1084

Falanga, M., Bonnet-Bidaud, J. M., Poutanen, J., et al. 2005a, A\&A, 436, 647

Falanga, M., Kuiper, L., Poutanen, J., et al. 2005b, A\&A, 444, 15

Falanga, M., Poutanen, J., Bonning, E. W., et al. 2007, A\&A, 464, 1069

Ford, E. C. 2000, ApJ, 535, L119

Frank, J., King A., Raine D. 2002, in Accretion Power in Astrophysics (Camb. Univ. Press)

Fujimoto, M. Y., Hanawa, T., \& Miyaji, S. 1981, ApJ, 247, 267

Fujimoto, M. Y., Sztajno, M., Lewin, W. H. G., \& van Paradijs, J. 1987, ApJ, 319,902

Galloway, D., \& Cumming, A. 2006, ApJ, 652, 559

Galloway, D. K., Cumming, A., Kuulkers, E., et al. 2004, ApJ, 601, 466

Gilfanov, M., Revnivtsev, M., Sunyaev, R., \& Churazov, E. 1998, A\&A, 338, L83

Gierliński, M., \& Poutanen, J. 2005, MNRAS, 359, 1261

Gierliński, M., Done, C., \& Barret, D. 2002, MNRAS, 331, 141

Goldwurm, A., David, P., Foschini, L., et al. 2003, A\&A, 411, L223

Gros, A., Goldwurm, A., Cadolle-Bel, M., et al. 2003, A\&A, 411, L179

Haardt, F., \& Maraschi, L. 1993, ApJ, 413, 507

Hartman, J. M., Patruno, A., Chakrabarty, D., et al. 2009, ApJ, 702, 1673

Hartman, J. M., Galloway, D. K., \& Chakrabarty, D. 2011, ApJ, 726, 26

Iacolina, M. N., Burgay, M., Burderi, L., et al. 2010, A\&A, 519, A13

Ibragimov, A., \& Poutanen, J. 2009, MNRAS, 400, 492

Ibragimov, A., Kajava, J., \& Poutanen, J. 2010, MNRAS, submitted

Inogamov, N. A., \& Sunyaev, R. A. 1999, Astron. Lett., 25, 269

Jahoda, K., Swank, J. H., Giles, A. B., et al. 1996, Proc. SPIE, 2808, 59

King, A. R., \& Ritter, H. 1998, MNRAS, 293, L42

Kluźniak, W., \& Wilson, J. R. 1991, ApJ, 372, L87

Kuiper, L., Hermsen, W., den Hartog, P. R., \& Collmar, W. 2006, ApJ, 645, 556

Kuulkers, E., den Hartog, P. R., in 't Zand, J. J. M., et al. 2003, A\&A, 399, 663

Lebrun, F., Leray, J.-P., Lavocate, Ph., et al. 2003, A\&A, 411, L141

Lewin, W. H. G. L., van Paradijs, J., \& Taam, R. 1993, Sp. Sci. Rev., 62, 223

Linares, M., van der Klis, M., \& Wijnands, R. 2007, ApJ, 660, 595
Lund, N., Budtz-Joergensen, C., Westgaard, N. J., et al. 2003, A\&A, 411, L231

Malzac, J., Beloborodov, A. M., \& Poutanen, J. 2001, MNRAS, 326, 417

Mitsuda, K., Inoue, H., Koyama, K., et al. 1984, PASJ, 36, 741

Markwardt, C. B., Altamirano, D., Swank, J. H., et al. 2009a, ATel, 2197

Markwardt, C. B., Altamirano, D., Strohmayer, T. E., \& Swank, J. H., 2009b, ATel, 2237

Miller-Jones, J. C. A., Russell, D. M., \& Migliari, S. 2009, ATel, 2232

Nowak, M. A., Paizis, A., Wilms, J., et al. 2009, ATel, 2215

Papitto, A., Riggio, A., Burderi, L., et al. 2009, ATel, 2220

Papitto, A., Riggio, A., Di Salvo, T., et al. 2010, MNRAS, 407, 2575

Papitto, A., Riggio, A., Burderi, L., et al., 2011, A\&A, 528, A55

Patruno, A. 2010, ApJ, 722, 909

Psaltis, D., \& Chakrabarty, D. 1999, ApJ, 521, 332

Poutanen, J. 2006, Adv. Space Res., 38, 2697

Poutanen, J. 2008, in A Decade of Accreting Millisecond X-ray Pulsars, ed. R. Wijnands, D. Altamirano, P. Soleri, N. Degenaar, N. Rea, P. Casella, A. Patruno, \& M. Linares (New York: AIP), AIP Conf. Ser., 1068, 77

Poutanen, J., \& Svensson, R. 1996, ApJ, 470, 249

Poutanen, J., \& Gierliński, M. 2003, MNRAS, 343, 1301

Poutanen, J., Ibragimov, A., \& Annala, M. 2009, ApJ, 706, L129

Powell, C. R., Haswell, C. A., \& Falanga, M. 2007, MNRAS, 374, 466

Riggio, A., Papitto A., Burderi, L., et al. 2009, ATel, 2221

Riggio, A., Papitto, A., Burderi, L., et al. 2011, A\&A, 526, A95

Rothschild, R. E., Blanco, P. R., Gruber, D. E., et al. 1998, ApJ, 496, 538

Stern, B. E., Poutanen, J., Svensson, R., et al. 1995, ApJ, 449, L13

Strohmayer, T., \& Bildsten, L. 2006, in Compact stellar X-ray sources, ed. W. H. G. Lewin, \& M. van der Klis (Cambridge: Cambridge University Press), 113

Strohmayer, T. E., Markwardt, C. B., Swank, J. H., \& in't Zand, J. 2003, ApJ, 596, L67

Suleimanov, V., \& Poutanen, J. 2006, MNRAS, 369, 2036

Thompson, T. W. J., Galloway, D. K., Rothschild, R. E., \& Homer, L. 2008, ApJ, 681,506

Torres, M. A. P., Jonker, P. G., Steeghs, D., Simon, J. D., \& Gutowski, G. 2009a, ATel, 2216

Torres, M. A. P., Jonker, P. G., Steeghs, D., et al. 2009b, ATel, 2233

Ubertini, P., Lebrun, F., Di Cocco, G., et al. 2003, A\&A, 411, L131

Viironen, K., \& Poutanen, J. 2004, A\&A, 426, 985

Watts, A. L., Strohmayer, T. E., \& Markwardt, C. B. 2005, ApJ, 634, 547

Watts, A. L., Altamirano, D., Markwardt, C. B., \& Strohmayer, T. E. 2009, ATel, 2199

Wijnands, R. 2006, in Trends in Pulsar Research, ed. J. A. Lowry (NY: Nova Science Publishers), 53

Wijnands, R., \& van der Klis, M. 1998, Nature, 394, 344

Winkler, C., Courvoisier, T. J.-L., Di Cocco, G., et al. 2003, A\&A, 411, L1 